\title{
Oncolytic herpes simplex virus armed with xenogeneic homologue of prostatic acid phosphatase enhances antitumor efficacy in prostate cancer
}

\author{
P Castelo-Branco $^{1,2,3}$, BJ Passer ${ }^{1,2}$, JS Buhrman ${ }^{1}$, S Antoszczyk ${ }^{1,2}$, M Marinelli ${ }^{1}$, C Zaupa ${ }^{1,2}$, SD Rabkin ${ }^{1,2}$ \\ and RL Martuza ${ }^{1,2}$ \\ ${ }^{1}$ Molecular Neurosurgery Laboratory, Department of Neurosurgery, Massachusetts General Hospital, Boston, MA, USA and ${ }^{2}$ Harvard \\ Medical School, Boston, MA, USA
}

\begin{abstract}
Prostate cancer is one of the most prevalent cancers in men. Replication-competent oncolytic herpes simplex virus (oHSV) vectors are a powerful antitumor therapy that can exert at least two effects: direct cytocidal activity that selectively kills cancer cells and induction of antitumor immunity. In addition, oHSV vectors can also function as a platform to deliver transgenes of interest. In these studies, we have examined the expression of a xenogeneic homologue of the prostate cancer antigen, prostatic acid phosphatase (PAP), with the goal of enhancing virotherapy against $P A P$-expressing tumors. $P A P$ has already been used for cancer vaccination in patients with prostate cancer. Here we show that treatment with oHSV bPA6 expressing
\end{abstract}

Keywords: HSV; TRAMP; virotherapy; cancer therapy

\section{Introduction}

Prostate cancer is the most common cancer among men in developed countries, and in the United States alone over 180000 new cases were diagnosed in $2008 .^{1}$ Treatment options for prostate cancer include surgery with radical prostatectomy, hormone therapy, chemotherapy $^{2}$ and radiation. ${ }^{3}$ However, severe secondary effects are common among patients and there is no curative treatment once the primary tumor metastasizes. ${ }^{4}$ The introduction of genetically engineered oncolytic viruses as a new therapeutic avenue in the battle against cancer began with the use of oncolytic herpes simplex viruses (oHSVs). ${ }^{5}$ Typically, oHSV carry mutations in the viral genome that enable them to replicate in and kill cancer cells, without harming normal tissue. ${ }^{6}$ Among the benefits of oHSV vectors are that they are easily manipulated, can carry transgene inserts ${ }^{7}$ and have

Correspondence: Dr SD Rabkin, Molecular Neurosurgery Laboratory, Massachusetts General Hospital, MGH-Simches Research Bldg, 185 Cambridge St, CPZN-3800, Boston, MA 02114, USA.

E-mail: rabkin@helix.mgh.harvard.edu

${ }^{3}$ Current address: Arthur and Sonia Labatt Brain Tumour Research Centre, Hospital for Sick Children, University of Toronto, Toronto, Ontario, Canada.

Received 27 September 2009; revised 5 January 2010; accepted 20 January 2010; published online 11 March 2010 xenogeneic human PAP (hPAP) significantly reduces tumor growth and increases survival of C57/BL6 mice bearing mouse TRAMP-C2 prostate tumors, whereas expression of syngeneic mouse PAP (MPAP) from the same oHSV vector did not enhance antitumor activity. Treatment of mice bearing metastatic TRAMP-C2 lung tumors with oHSV-expressing hPAP resulted in fewer tumor nodules. To our knowledge, this is the first report of oncolytic viruses being used to express xenoantigens. These data lend support to the concept of combining oncolytic and immunogenic therapies as a way to improve therapy of metastatic prostate cancer.

Gene Therapy (2010) 17, 805-810; doi:10.1038/gt.2010.20; published online 11 March 2010 already been safely used in human subjects with a variety of cancers. ${ }^{8,9}$ Another advantage of oHSV is that it can interact synergistically with other therapeutic modalities, including chemotherapeutic agents ${ }^{10,11}$ and radiotherapy, ${ }^{12}$ thereby promoting better therapeutic outcomes by targeting more then one aspect of cancer biology.

Previous work from our laboratory and others has shown that oHSV san be used as an in situ vaccine to generate a tumor-specific host immune response. ${ }^{13-16}$ Prostatic acid phosphatase (PAP) is a prostate-specific antigen that is expressed in both prostate cells and prostate cancer cells ${ }^{17}$ as well as other adenocarcinomas. ${ }^{18}$ However, as it is a self-antigen it is not immunogenic due presumably to tolerance. It has previously been shown that immunization of rats with human PAP (hPAP) generates a cytotoxic T lymphocyte response leading to tissue-specific prostatitis. ${ }^{19}$ Furthermore, immunization of patients with metastatic prostate cancer with recombinant mouse PAP (mPAP) loaded dendritic cells has been shown to result in an antitumor immune response and clinical stabilization of the disease, as indicated by a decreased rise of serum prostatespecific antigen levels. ${ }^{18}$ This suggests that vaccination with a xenogeneic homolog can break tolerance to a self-antigen, hPAP, inducing a Th1-type cytokine response to both mPAP and hPAP antigens. Other studies have confirmed this concept of using a xenogenic form of an antigen to break antitumor tolerance to other proteins, such as 
prostate stem cell antigen, where DNA vaccination induced antitumor immune responses against TRAMP-C1 tumors, ${ }^{19}$ prostate-specific membrane antigen, ${ }^{20}$ gp100 in melanoma, ${ }^{21}$ HER2 expressed from a replication-deficient adenovirus vector in breast cancer ${ }^{22}$ and epidermal growth factor receptor. $^{23}$ We therefore hypothesized that an oHSV expressing a xenogenic PAP could be used to treat prostate cancer.

In this study, we have combined the ability of oHSV to specifically replicate in and kill cancer cells with the immunogenic effects of in situ vaccination with a xenogeneic PAP protein to create a more powerful therapeutic strategy for prostate cancer. Our results show that this combined approach achieves improved inhibition of established tumor growth and animal survival compared to oHSV alone or when expressing syngeneic mPAP. These results represent an encouraging step toward the development of a viable therapy for prostate cancer and describe a novel approach that should be applicable to other oncolytic viruses.

\section{Results and Discussion}

\section{Characterization of oHSVs expressing MPAP and hPAP transgenes}

To construct oHSVs expressing hPAP (bP $\Delta 6$-hPAP) or the mouse homolog (bP $\Delta 6$-mPAP), we used the Flip-Flop HSV-BAC technology. ${ }^{7}$ A third construct, lacking a transgene (bP $\Delta 6$-empty), was also generated to be used as a control (Supplementary Figure 1). The backbone for these vectors was HSV-1 strain Patton, from which P $\Delta 6$ BAC was constructed by inserting the BAC cassette into the ICP6 (UL39) gene, as previously described. ${ }^{7}$ The genomic structure of the vectors was confirmed by restriction endonuclease digestion and gel electrophoresis and the recombinant vectors all express $\beta$-galactosidase, which is detectable after X-gal histochemistry (data not shown). Although mouse prostate cancer cells are much less susceptible to oHSV replication than human prostate cancer cells (compare Walker et $a l^{24}$ and Varghese et $a l^{25}$ ) or Vero cells, they still replicate and spread in mouse TRAMP-C2 and RM-1 prostate cancer cells (Figure 1a). The transgene expressing viruses replicate less well than the parental wild-type strain Patton (WT, Figure 1b), likely due to the ICP6 mutation; however, there is no difference between bP $\Delta 6$-hPAP and bP $\Delta 6$-empty (Figure $1 \mathrm{~b}$ ). A dose-response curve for killing TRAMP-C2 cells is shown in Figure 1c. Again there is no difference between bP $\Delta 6$-hPAP and bP $\Delta 6$ empty, showing that expression of hPAP does not alter the in vitro replication or cytotoxicity of $\mathrm{bP} \Delta 6$.

Next we confirmed that the viruses express the inserted transgenes. The cytomegalovirus immediate early promoter was used to propel transgene expression, as we have described before, ${ }^{26,27}$ because it provides high level expression. Vero cells were infected with either bP $\Delta 6$-hPAP, bP $\Delta 6$-mPAP or bP $\Delta 6$-empty and RT-PCR
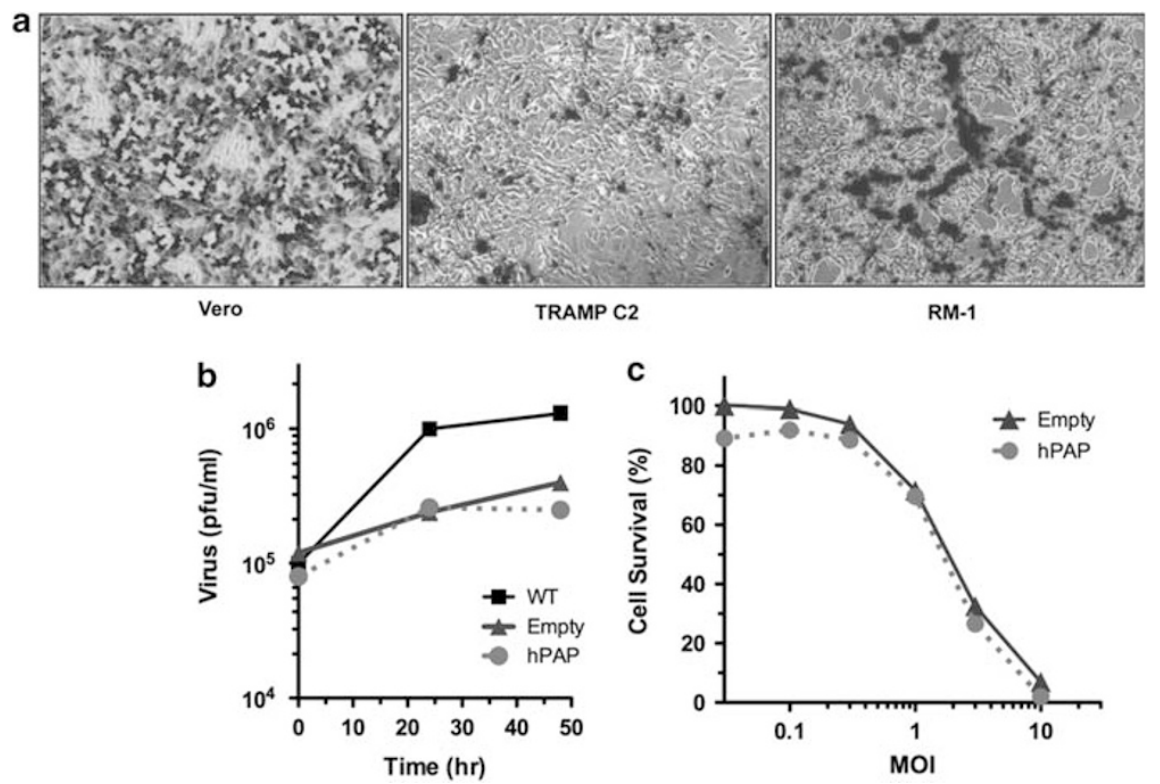

Figure 1 Characterization of bP $\Delta 6$ transgene vectors. (a) bP $\Delta 6$-hPAP viruses infect and spread in Vero and mouse prostate cancer cells. Vero (African Green Monkey Kidney) cells (American Type Culture Collection, Manassas, VA, USA) were cultured in Dulbecco's modified Eagle's medium (DMEM) with glucose (4.5 $\mathrm{g}^{-1}$; Mediatech Inc., Herndon, VA, USA) supplemented with $10 \%$ fetal calf serum (FCS). TRAMP-C2,28,34 obtained from Dr N Greenberg (Fred Hutchinson Cancer Research Center, Seattle, WA, USA), and RM- $1,{ }^{35}$ obtained from Dr T C Thompson (Baylor College of Medicine, Houston, TX, USA), were cultured as previously described. Cells were seeded at $80 \%$ confluency and $24 \mathrm{~h}$ later infected with bP $\triangle 6$-hPAP at a multiplicity of infection (MOI) of 1. Cultures were fixed $18 \mathrm{~h}$ after infection and stained for $\beta$-galactosidase expression with X-Gal $\left(1 \mathrm{mg} \mathrm{ml}^{-1}\right), 5 \mathrm{mM}$ potassium ferricyanide, $5 \mathrm{mM}$ potassium ferrocyanide, $2 \mathrm{mM}$ magnesium chloride in phosphatebuffered saline (PBS) for $4 \mathrm{~h}$ at $37^{\circ} \mathrm{C}$. Cells were washed with PBS, counterstained with neutral red solution and $\beta$-galactosidase expression and viral spread visualized under a light microscope. (b) Virus replication assays. Vero cells were seeded at $1 \times 10^{5}$ cells per well in 12 -well plates and $24 \mathrm{~h}$ later infected with either $\mathrm{bP} \Delta 6-\mathrm{hPAP}, \mathrm{bP} \Delta 6$-empty or wt viruses at $\mathrm{MOI}=1$. At $2 \mathrm{~h}$ after infection the inoculum was removed and replaced with medium (DMEM/1\% inactivated FCS). Cells and medium were harvested at indicated times after infection, processed with freeze/thaw cycles and sonication, and titered on Vero cells. Virus yield is plotted as plaque-forming units (PFU) per ml. (c) Cell viability assays. TRAMP-C2 cells were seeded into 96 -well plates at 5000 cells per well and $24 \mathrm{~h}$ later infected with bP $\Delta 6$-hPAP $\left(\mathrm{IC} \mathrm{C}_{50}=2.5\right)$ or $\mathrm{bP} \Delta 6$-empty $\left(\mathrm{IC}_{50}=2.9\right)$ at threefold serial dilutions (from 0.01 to $10 \mathrm{PFU}$ per cell). Cell viability was assessed 4 days after infection with 3-(4,5-dimethylthiazol-2-yl)-2,5-diphenyltetrazolium bromide (Sigma, St Louis, MO, USA) according to the manufacturer's instructions. 

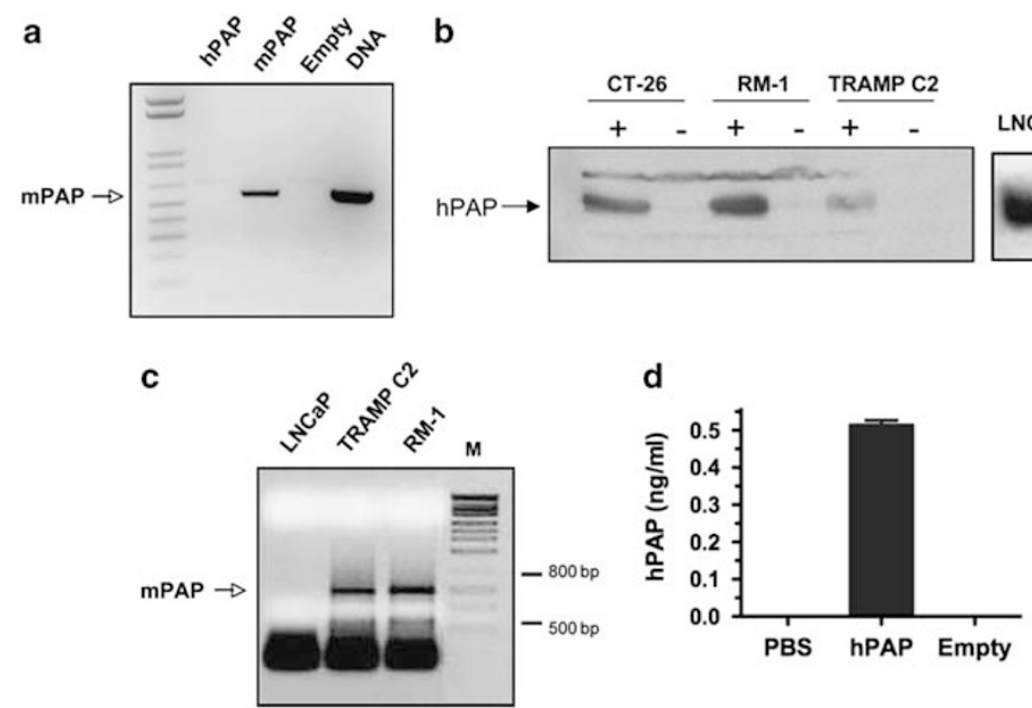

Figure 2 Prostatic acid phosphatase (PAP) expression. (a) Reverse transcriptase (RT)-PCR analysis of Vero cells infected with bP $\Delta 6$-mPAP shows mouse PAP (mPAP) mRNA expression. Cells were grown in six-well plates and infected at 90-95\% confluence with either bP $\Delta 6$-hPAP, bP $\Delta 6$-mPAP or bP $\Delta 6$-empty viruses at a multiplicity of infection (MOI) of 2 . After $2 \mathrm{~h}$, virus inoculum was removed and replaced with medium Dulbecco's modified Eagle's medium (DMEM) $/ 1 \%$ inactivated fetal calf serum (FCS). After $8 \mathrm{~h}$, the total RNA fraction was isolated from cell lysates using TRIzol (Invitrogen, Carlsbad, CA, USA), and treated with RQ1 RNase-free DNase I (Promega, Madison, WI, USA). Reverse transcription was carried out using SuperScriptIII First Strand Synthesis kit (Invitrogen) and random hexamer primers. cDNAs were subjected to conventional PCR $\left(5 \mathrm{~min}\right.$ at $95{ }^{\circ} \mathrm{C}$ then 28 cycles: $30 \mathrm{~s}$ at $95{ }^{\circ} \mathrm{C}, 15 \mathrm{~s}$ at $68{ }^{\circ} \mathrm{C}, 20 \mathrm{~s}$ at $72{ }^{\circ} \mathrm{C}$; and terminal elongation 2 min at $72{ }^{\circ} \mathrm{C}$ ) with specific primers, P1 mPAP619 $5^{\prime}$-gcttcctggacaccttgtcgtcgctgtcg-3' and P2 mPAP1214 $5^{\prime}$-attccgtccttggtggctgc- $3^{\prime}$, designed to generate a PCR product of $595 \mathrm{bp}$. As a positive control, plasmid DNA (pVec92-mPAP) was used and the PCR was performed without the reverse transcription step. The reaction product was analyzed by $1.2 \%$ agarose gel electrophoresis and stained with ethidium bromide. (b) Western blot analysis of hPAP expression in CT-26 colorectal carcinoma, ${ }^{13}$ RM-1 and TRAMP-C2 cell lines after infection with bP $\Delta 6$-hPAP (indicated by + ) or bP $\Delta 6$-empty (indicated by - ) at MOI $=2$. Human prostate adenocarcinoma LNCaP cells ${ }^{24}$ and human embryonic kidney 293 cells were used as controls. Cell lysates were prepared using RIPA buffer ${ }^{36} 24 \mathrm{~h}$ after infection. Each lysate was separated by SDS-polyacrylamide electrophoresis, transferred to polyvinylidene fluoride (PVDF) membrane (Bio-Rad, Hercules, CA, USA), and immunoblotted with anti-hPAP (Dako, Carpinteria, CA, USA) Rabbit anti-human PAP, A0627) antibody (diluted 1:1000) using standard procedures. (c) Mouse prostate cancer cells express PAP. Total RNA was isolated from LNCaP, TRAMP-C2 and RM-1 cells and RT-PCR was performed using primers previously described. $^{37}$ mPAP mRNA was observed in both TRAMP-C2 and RM-1 cells but not in human LNCaP prostate cancer cells. (d) In vivo expression of hPAP. TRAMP-C2 cells $\left(5 \times 10^{6}\right.$ per mouse) were implanted subcutaneously into the flanks of male 6 - to 8 -week-old C57/BL6

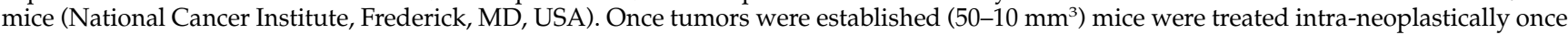
with bP $\triangle 6$-hPAP $\left(2 \times 10^{7} \mathrm{PFU}\right)$. After $48 \mathrm{~h}$, tumors were harvested, homogenized and centrifuged. Supernatants were assayed for hPAP using an enzyme-linked immunosorbent assay (ELISA) kit (R\&D Systems, Minneapolis, MN, USA) according to manufacturer's instructions. Error bars represent the standard deviation of three measurements. All in vivo procedures were approved by the Massachusetts General Hospital Subcommittee on Research Animal Care.

analysis was carried out for mPAP, using mouse transgene-specific primers, and a band corresponding to the expected mPAP RNA was only seen in bP $\Delta 6-$ mPAP-infected Vero cells (Figure 2a). Expression of the hPAP transgene in mouse tumor cell lines was detected with human-specific anti-PAP antibody. Western blot analysis reveals a band corresponding to hPAP in all lanes where cells were infected with bP $\Delta 6$-hPAP (+, Figure 2b). As a control, we show the expression of endogenous hPAP in human LNCaP prostate cells but not in 293 cells (Figure 2b, right). To test the immunogenic effects of the oHSV PAP vectors, it was necessary that the mouse prostate tumor model expressed mPAP. RT-PCR analysis confirmed the presence of mPAP RNA in both TRAMP-C2 and RM-1 prostate cancer cells (Figure 2c). Ultimately, TRAMP-C2 was selected as our experimental tumor cell line because previous data from our lab has shown that these cells are responsive to oHSV treatment in both primary and metastatic tumor models. ${ }^{25,28}$ Finally, to verify that bP $\Delta 6$-hPAP expresses hPAP protein in vivo, we implanted TRAMP-C2 cells subcutaneously into C57/BL6 immunocompetent mice. Once tumors were established, the mice were injected intra-neoplastically with bP $\Delta 6$-hPAP or bP $\Delta 6$-empty. At $48 \mathrm{~h}$ after injection, hPAP was detected in tumors treated with bP $\Delta 6$-hPAP, but not with bP $\Delta 6$-empty (Figure $2 \mathrm{~d}$ ).

\section{In vivo treatment with $b P \Delta 6-h P A P$ leads to tumor reduction and increased animal survival}

TRAMP-C2 cells were implanted subcutaneously in syngeneic C57/BL6 mice. Once tumors were established, mice were randomized into four treatment groups: $b P \Delta 6-$ hPAP, bP $\Delta 6$-mPAP, bP $\Delta 6$-empty and phosphate-buffered saline (PBS) only. Each mouse was treated four times intra-neoplastically with $1 \times 10^{7}$ plaque-forming units (PFU) per injection over a period of 10 days and then monitored for tumor growth and survival. Mice treated with oHSV lacking transgene (bP $\Delta 6$-empty) or oHSV expressing a non-xenogenic form of PAP (bP $\Delta 6-\mathrm{mPAP})$ showed a slower rate of tumor growth, with no difference between mPAP and empty $(P=0.7)$ and not significantly different from the PBS-treated mice (PBS vs mPAP $P=0.09$, PBS vs empty $P=0.08$ ). However, mice treated with the xenogenic hPAP-expressing oHSV (bP $\Delta 6$-hPAP) showed a significant inhibition in 

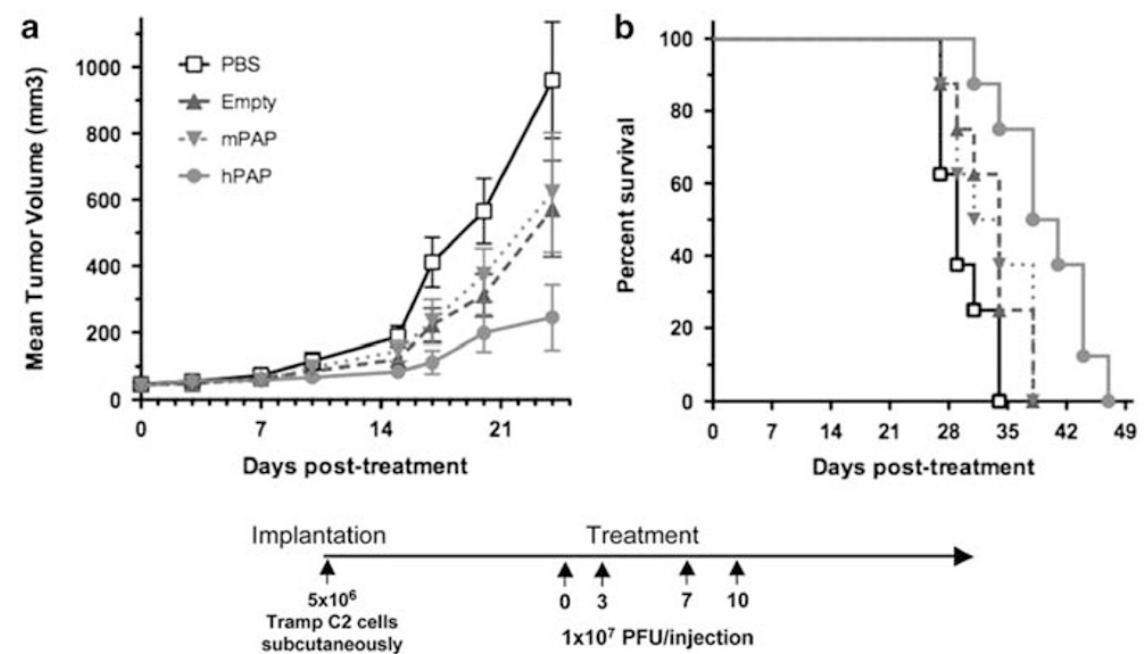

Figure 3 Mice treated with bP $\triangle 6$-hPAP show tumor reduction and increased survival. C57/BL6 mice were implanted subcutaneously in the flanks with TRAMP-C2 cells $\left(5 \times 10^{6}\right.$ per mouse). Once tumors were established $\left(50-100 \mathrm{~mm}^{3}\right)$ mice $(N=8)$ were randomized and treated four times with $1 \times 10^{7} \mathrm{PFU}$ of bP $\Delta 6$-empty, bP $\Delta 6$-mPAP, bP $\Delta 6$-hPAP or phosphate-buffered saline (PBS) over a period of 10 days by direct intratumoral inoculation. Tumor volumes were monitored using calipers and calculated with the formula $v=\left[(\right.$ length $) \times\left(\right.$ width $\left.\left.{ }^{2}\right)\right] / 2$. Mice were killed when tumors reached $1000 \mathrm{~mm}^{3}$. Animals treated with $\mathrm{bP} \Delta 6$-hPAP showed a significant reduction in tumor volume $(P=0.002 ; \mathrm{PBS}$ vs hPAP, $P=0.04$; empty vs hPAP, $P=0.03$; mPAP vs hPAP) (a) and a significant increase in survival (b) when compared with the bP $\Delta 6$-mPAP $(P=0.01)$, bP $\Delta 6$-empty $(0.01)$ or PBS $(P=0.0008)$ groups. Analysis of tumor volumes (day 24, Student's $t$-test) and survival (log-rank test) was performed with GraphPad Prism v.4 (San Diego, CA, USA).

tumor growth when compared to bP $\triangle 6$-mPAP $(P=0.03)$, bP $\Delta 6$-empty $(P=0.04)$ or PBS $(P=0.002)$ groups (Figure 3a). This inhibition translated into a significant increase in survival (Figure $3 \mathrm{~b}$ ) when compared to mice treated with bP $\triangle 6$-mPAP $(P=0.01)$, bP $\Delta 6$-empty $\quad(P=0.01)$ or PBS only $(P=0.0008)$, with the median survival increasing from 29 days for PBS to 39 days for hPAP. In a separate experiment, three intra-tumoral injections of bP $\triangle 6$-hPAP resulted in significantly extended survival compared to bP $\Delta 6$-empty or PBS (data not shown). This indicates that expression of xenogenic hPAP is enhancing antitumor activity.

\section{Systemically administrated $b P \Delta 6-h P A P$ reduces tumor nodule burden in a metastatic tumor model}

To investigate the therapeutic efficacy of bP $\Delta 6$-hPAP in a metastatic tumor model, we injected TRAMP-C2 cells intravenously through the tail vein. TRAMP-C2 cells metastasize primarily to the lungs. ${ }^{28}$ Tumor-bearing mice were systemically treated through tail vein injection with bP $\triangle 6$-hPAP, bP $\Delta 6$-mPAP, bP $\Delta 6$-empty or PBS four times over a period of 10 days. Mice were killed at day 60 and the lungs were stained with India ink to detect lung tumor nodules, which were measured and counted. Mice treated with bP $\triangle 6$-hPAP showed significantly fewer tumors per group than the bP $\Delta 6$-empty $(P=0.015)$ or PBS $(P=0.017)$ groups and also a significant reduction in tumor burden when compared with bP $\Delta 6$-empty $(P=0.034)$ (Table 1$)$.

In an effort to improve the therapeutic potential of the oHSV vector system, we combined oHSV with a xenoantigen transgene, designed to stimulate an autoimmune response against a prostate antigen, PAP. For these studies, we developed a new oHSV vector using wild-type HSV-1 strain Patton as the backbone and FlipFlop HSV-BAC technology ${ }^{7}$ to insert the PAP transgenes
Table $1 \mathrm{bP} \Delta 6$-hPAP treatment leads to fewer tumor nodules in a metastatic tumor model

PBS Empty mPAP hPAP

$\begin{array}{lllll}\text { Total tumor nodules } & 8 & 18 & 10 & 4^{*} \\ \text { Mean tumor burden per mouse }\left(\mathrm{cm}^{2}\right) & 7.0 & 16.8 & 6.9 & 1.6^{* *}\end{array}$

TRAMP-C2 cells $\left(5 \times 10^{5}\right.$ per mouse $)$ were injected intravenously in the tail vain of C57/BL6 mice ( $N=8$ per group) and lung tumors established as previously described. ${ }^{28}$ Preliminary studies showed microscopic tumor nodules by day 25 and by day 60 animals showed signs of morbidity. Mice were treated four times with $3 \times 10^{7}$ PFU of bP $\Delta 6$-hPAP, bP $\Delta 6$-mPAP or bP $\Delta 6$-empty, or PBS over a period of 10 days. Animals were killed at day 60 and the number of lung tumor nodules and tumor burden per mouse was analyzed as described previously. ${ }^{28}$ Animals treated with bP $\Delta 6$-hPAP viruses showed significantly fewer tumor nodules when compared with bP $\triangle 6$-mPAP or PBS groups $\left({ }^{*} P=0.02\right)$, as well as a decrease in tumor burden $(* * P=0.03$ vs bP $\Delta 6$-empty). There was no statistical difference between tumor nodules in the bP $\Delta 6$-empty vs PBS groups. Pairwise statistical analysis were performed using Student's $t$-tests.

into the ICP6 locus of the oHSV genome in a rapid manner. Many oHSV vectors contain ICP6 mutations, which inactivate viral ribonucleotide reductase, impeding virus replication in normal quiescent cells except those lacking p16, such as many tumor cells. ${ }^{29} \mathrm{HrR3}$, with an insertion of Escherichia coli LacZ inactivating ICP6 in the HSV-1 KOS backbone was the first such vector. ${ }^{30,31} \mathrm{bP} \Delta 6$ has the same LacZ insertion into ICP6 as hrR3, but in a Patton backbone. Expression of PAP did not affect virus replication or tumor cell killing in vitro. To test the xenoantigen strategy, we needed a tumor model with endogenous mPAP expression that progressed slowly enough to allow the treated animals time to generate an immune response, and that was susceptible to oHSV. For these reasons we chose the TRAMP-C2 
tumor cell line. Incorporating hPAP transgene into the oHSV vector bP $\Delta 6$ led to a reduction in tumor burden in subcutaneous and metastatic prostate tumor models that did not occur with mPAP. Because of limited oHSV replication in mouse prostate cancer cells, syngeneic mouse prostate tumors have been difficult to treat with oHSV, except with the addition of immune-modulatory transgenes, such as IL-12. ${ }^{25,28}$

Our data support the concept that better outcomes can be achieved by combining oncolytic viral therapy with immunotherapy than with oncolytic viral therapy alone. The ability of hPAP, but not mPAP, to enhance the antitumor activity of oHSV indicates that a specific immune response is at work, engendered by exposure to the xenogeneic PAP protein and resulting in a loss of tolerance to syngeneic PAP. Previous studies vaccinating rats with vaccinia virus or plasmid-expressing xenogeneic hPAP transgenes showed the induction of a major histocompatibility class I-dependent cytotoxic T lymphocytemediated anti-PAP autoimmune response, but not an antibody response. ${ }^{32,33}$ In these studies, the response differed to some extent between rat strains and the animals did not bear prostate tumors. To our knowledge, ours is the first report of an oncolytic virus expressing a xenoantigen for cancer vaccination. It is likely that the combination of hPAP with expression of other immunomodulatory molecules will further improve the efficacy of oHSV-mediated immunotherapy. As we have shown that oHSV-expressing hPAP is efficacious in treating mouse prostate cancer, we hypothesize that oHSVexpressing mPAP would be effective in treating human prostate cancer. This could be addressed in a clinical trial for the treatment of prostate cancer.

\section{Conflict of interest}

The authors declare no conflict of interest.

\section{Acknowledgements}

We thank Dr M Crew for providing the mPAP cDNA and Dr Timothy C. Thompson for providing RM-1 cells. This work was supported in part by grants to RLM from the DOD (W81XWH-04-1-0254) and the NIH (R01 CA102139).

\section{References}

1 Moul JW, Mouraviev V, Sun L, Schroeck FR, Polascik TJ. Prostate cancer: the new landscape. Curr Opin Urol 2009; 19: 154-160.

2 Mitchell RE, Chang SS. Current controversies in the treatment of high-risk prostate cancer. Curr Opin Urol 2008; 18: 263-268.

3 Sia M, Rosewall T, Warde P. Radiotherapy as primary treatment modality. Front Radiat Ther Oncol 2008; 41: 15-25.

4 Kumar-Sinha C, Tomlins SA, Chinnaiyan AM. Recurrent gene fusions in prostate cancer. Nat Rev Cancer 2008; 8: 497-511.

5 Martuza RL, Malick A, Markert JM, Ruffner KL, Coen DM. Experimental therapy of human glioma by means of a genetically engineered virus mutant. Science 1991; 252: 854-856.

6 Varghese S, Rabkin SD. Oncolytic herpes simplex virus vectors for cancer virotherapy. Cancer Gene Ther 2002; 9: 967-978.

7 Kuroda T, Martuza RL, Todo T, Rabkin SD. Flip-Flop HSV-BAC: bacterial artificial chromosome based system for rapid generation of recombinant herpes simplex virus vectors using two independent site-specific recombinases. BMC Biotech 2006; 6: 40.

8 Aghi M, Martuza RL. Oncolytic viral therapies-the clinical experience. Oncogene 2005; 24: 7802-7816.

9 Liu TC, Galanis E, Kirn D. Clinical trial results with oncolytic virotherapy: a century of promise, a decade of progress. Nat Clin Pract Oncol 2007; 4: 101-117.

10 Post DE, Fulci G, Chiocca EA, Van Meir EG. Replicative oncolytic herpes simplex viruses in combination cancer therapies. Curr Gene Ther 2004; 4: 41-51.

11 Passer BJ, Castelo-Branco P, Buhrman JB, Varghese S, Rabkin SD, Martuza RL. Oncolytic herpes simplex virus vectors and taxanes synergize to promote killing of prostate cancer cells. Cancer Gene Ther 2009; 16: 551-560.

12 Harrington KJ, Melcher A, Vassaux G, Pandha HS, Vile RG. Exploiting synergies between radiation and oncolytic viruses. Curr Opin Mol Ther 2008; 10: 362-370.

13 Toda M, Rabkin SD, Kojima H, Martuza RL. Herpes simplex virus as an in situ cancer vaccine for the induction of specific anti-tumor immunity. Hum Gene Ther 1999; 10: 385-393.

14 Thomas DL, Fraser NW. HSV-1 therapy of primary tumors reduces the number of metastases in an immune-competent model of metastatic breast cancer. Mol Ther 2003; 8: 543-551.

15 Benencia F, Courrèges MC, Conejo-García JR, Mohamed-Hadley A, Zhang L, Buckanovich RJ et al. HSV oncolytic therapy upregulates interferon-inducible chemokines and recruits immune effector cells in ovarian cancer. Mol Ther 2005; 12: 780-802.

16 Li H, Dutuor A, Tao L, Fu X, Zhang X. Virotherapy with a type 2 herpes simplex virus-derived oncolytic virus induces potent antitumor immunity against neuroblastoma. Clin Cancer Res 2007; 13: 316-322.

17 Lin MF, DaVolio J, Garcia-Arenas R. Expression of human prostatic acid phosphatase activity and the growth of prostate carcinoma cells. Cancer Res 1992; 52: 4600-4607.

18 Fong L, Brockstedt D, Benike C, Breen JK, Strang G, Ruegg CL et al. Dendritic cell-based xenoantigen vaccination for prostate cancer immunotherapy. J Immunol 2001; 167: 7150-7156.

19 Ahmad S, Casey G, Sweeney P, Tangney M, O'Sullivan G. Prostate stem cell antigen DNA vaccination breaks tolerance to self-antigen and inhibits prostate cancer growth. Mol Ther 2009; 17: 1101-1108.

20 Gregor PD, Wolchok JD, Turaga V, Latouche JB, Sadelain M, Bacich D et al. Induction of autoantibodies to syngeneic prostatespecific membrane antigen by xenogeneic vaccination. Int $J$ Cancer 2005; 116: 415-421.

21 Gold JS, Ferrone CR, Guevara-Patiño JA, Hawkins WG, Dyall R, Engelhorn ME et al. A single heteroclitic epitope determines cancer immunity after xenogeneic DNA immunization against a tumor differentiation antigen. J Immunol 2003; 170: 5188-5194.

22 Gallo P, Dharmapuri S, Nuzzo M, Maldini D, Iezzi M, Cavallo F et al. Xenogeneic immunization in mice using HER2 DNA delivered by an adenoviral vector. Int J Cancer 2005; 113: 67-77.

23 Lu Y, Wei Y, Tian L, Zhao X, Yang L, Hu B et al. Immunogene therapy of tumors with vaccine based on xenogeneic epidermal growth factor receptor. J Immunol 2003; 170: 3162-3170.

24 Walker JR, McGeagh KG, Sundaresan P, Jorgensen TJ, Rabkin SD, Martuza RL. Local and systemic therapy of human prostate adenocarcinoma with the conditionally replicating herpes simplex virus vector G207. Hum Gene Ther 1999; 10: 2237-2243.

25 Varghese S, Rabkin SD, Liu R, Nielsen PG, Ipe T, Martuza RL. Enhanced therapeutic efficacy of IL-12, but not GM-CSF, expressing oncolytic herpes simplex virus for transgenic mouse derived prostate cancers. Cancer Gene Ther 2006; 13: 253-265.

26 Fukuhara H, Ino Y, Kuroda T, Martuza RL, Todo T. Triple genedeleted oncolytic herpes simplex virus vector double-armed with interleukin 18 and soluble B7-1 constructed by bacterial artificial chromosome-mediated system. Cancer Res 2005; 65: 10663-10668. 
27 Liu TC, Zhang T, Fukuhara H, Kuroda T, Todo T, Martuza RL et al. Oncolytic HSV armed with platelet factor 4, an antiangiogenic agent, shows enhanced efficacy. Mol Ther 2006; 14: 789-797.

28 Varghese S, Rabkin SD, Nielsen PG, Wang W, Martuza RL. Systemic oncolytic herpes virus therapy of poorly immunogenic prostate cancer metastatic to lung. Clin Cancer Res 2006; 12: 2919-2927.

29 Aghi M, Visted T, Depinho RA, Chiocca EA. Oncolytic herpes virus with defective ICP6 specifically replicates in quiescent cells with homozygous genetic mutations in p16. Oncogene 2008; 27: 4249-4254.

30 Goldstein DJ, Weller SK. Herpes simplex virus type 1-induced ribonucleotide reductase activity is dispensable for virus growth and DNA synthesis: isolation and characterization of an ICP6 lacZ insertion mutant. J Virol 1988; 62: 196-205.

31 Mineta T, Rabkin SD, Martuza RL. Treatment of malignant gliomas using ganciclovir-hypersensitive, ribonucleotide reductase-deficient herpes simplex viral mutant. Cancer Res 1994; 54: 3963-3966.

32 Fong L, Ruegg CL, Brockstedt D, Engleman EG, Laus R. Induction of tissue-specific autoimmune prostatitis with prostatic acid phosphatase immunization: implications for immunotherapy of prostate cancer. I Immunol 1997; 159: 3113-3117.

33 Johnson LE, Frye TP, Chinnasamy N, Chinnasamy D, McNeel DG. Plasmid DNA vaccine encoding prostatic acid phosphatase is effective in eliciting autologous antigen-specific CD8+ T cells. Cancer Immunol Immunother 2007; 56: 885-895.

34 Foster BA, Gingrich JR, Kwon ED, Madias C, Greenberg NM. Characterization of prostatic epithelial cell lines derived from transgenic adenocarcinoma of the mouse prostate (TRAMP) model. Cancer Res 1997; 57: 3325-3330.

35 Hall SJ, Mutchnik SE, Chen S-H, Woo SLC, Thompson TC. Adenovirus-mediated herpes simplex virus thymidine kinase gene and ganciclovir therapy leads to systemic activity against spontaneous and induced metastasis in an orthotopic mouse model of prostate cancer. Int J Cancer 1997; 70: 183-187.

36 Davis L, Kuehl M, Battey J. Basic Methods in Molecular Biology. Appleton \& Lange: Norwalk, CT, 1994.

37 Grossmann ME, Wood M, Celis E. Expression, specificity and immunotherapy potential of prostate-associated genes in murine cell lines. World J Urol 2001; 19: 365-370.

38 Vihko P, Virkkunen P, Henttu P, Roiko K, Solin T, Huhtala ML. Molecular cloning and sequence analysis of cDNA encoding human prostatic acid phosphatase. FEBS Lett 1988; 236: 275-281.

Supplementary Information accompanies the paper on Gene Therapy website (http://www.nature.com/gt) 